\title{
La mediación comunitaria en Brasil como instrumento de eficacia de la democracia participativa
}

\section{Community mediation in Brazil as a tool for effective participatory democracy}

\author{
Mônica Carvalho Vasconcelos
}

\section{Resumen}

El presente estudio tiene como objetivo principal evaluar la aplicabilidad de la mediación comunitaria en Brasil como instrumento de eficacia de la democracia participativa a través de un estudio predominantemente jurídico y bibliográfico sobre este mecanismo interdisciplinar de solución de conflictos. Es decir, se pretende investigar las consecuencias de la introducción de la mediación en las comunidades, sobretodo de baja renta, donde se constata un déficit de eficacia del derecho fundamental de acceso a la justicia. Esta investigación fue estructurada en tres partes distintas. La primera aborda el sentido de comunidad y sus peculiaridades. La segunda, a su vez, trata de la mediación en la comunidad como herramienta de empoderamiento individual y social. Por fin, el tercer apartado investiga la relevancia democratica de la Mediación Comunitaria en el escenario brasileño. No obstante la falta de ley específica sobre mediación, las experiencias prácticas se multiplican en el país, principalmente con la modalidad de mediación comunitaria. De esta suerte, el panorama actual de este mecanismo de solución de conflictos apunta para un creciente desarrollo que tiene apoyo de organizaciones nacionales e internacionales, gubernamentales $\mathrm{o}$ no, universidades, profesionales especializados de diferentes áreas de conocimiento, que han dispensado esfuerzos mutuos para resaltar la importancia de la mediación como sistema complementario de administración de justicia para lograr soluciones pacíficas, por medio del diálogo colaborativo.

Palabras-clave: Comunidad. Mediación. Democracia.

Doutora em Direitos Fundamentais pela Universidad Autónoma de Madrid. Coordenadora do Núcleo de Pesquisa do Centro de Ciências Jurídicas e professora de Direito Civil e Soluções Extrajudiciais de conflitos da Universidade de Fortaleza. Fortaleza - CE - Brasil. E-mail: mvasconcelos@unifor.br 


\section{Abstract}

This paper attempts to evaluate the applicability of community mediation in Brazil as a tool for effective participatory democracy through a predominantly legal and interdisciplinary literature review about this interdisciplinary mechanism. It means to investigate the consequences of the introduction of mediation in communities, especially low-income, where the people have many troubles to enforce their fundamental right of access to justice. This article was structured into three distinct parts. The first deals with the sense of community. The second, in turn, is about the mediated in the community as a tool for personal and social empowerment. Finally, the third section investigates the democratic relevance of Community Mediation in Brazil. This country doesn't have a specific law on mediation, but practical experiences are multiplied, mainly in the form of community mediation. In this way, the current situation of dispute resolution mechanism points to a growing development that has support from national and international specialized professionals from different areas of knowledge, they have shown mutual efforts to highlight the importance of mediations as a complementary system of justice to achieve peaceful solutions, through collaborative dialogue.

Keywords: Community. Mediation. Democracy.

\section{Introducción}

Existe en la actualidad un movimiento universal para hacer efectivo el acceso a la justicia, pues los sistemas jurídicos de gran parte de las sociedades occidentales no han sido adecuados para resolver los conflictos individuales y colectivos que se vuelven cada vez más intensos y se multiplican.

De hecho, numerosos estudios han constatado la grave crisis que afecta a la administración de la justicia de diversos países. En Brasil, las soluciones para enfrentar estas dificultades son complejas y exigen profundos cambios estructurales. La reforma de la justicia ha de hacerse globalmente, valiéndose de todos los medios legales, materiales y personales que están a disposición. En este contexto, los mecanismos complementarios de solución de conflictos están llamados a convertirse en pieza clave del nuevo modelo de justicia. 
Sin embargo, no se puede contemplar estos mecanismos sólo como una posibilidad de rescatar al colapsado Poder Judicial del país. Los resultados de distintas experiencias prácticas han demostrado que su aplicabilidad contribuye a la administración de justicia, pero esta no es su finalidad primordial. En verdad, la búsqueda de estas prácticas en las últimas décadas también deriva de la introducción de valores nuevos en la sociedad, de la ampliación de los espacios democráticos que repercute en la ciudadanía y exige posturas más participativas de la población en todos los niveles sociales.

Este estudio tiene como foco la mediación como proceso de intervención en la solución de conflictos que complementa la administración de justicia e introduce en la sociedad la cultura del diálogo y de la participación. Se trata de una temática actual y relevante en el mundo global y multicultural que vivimos, que presupone la negociación en todo instante de nuestras diferencias. Esta técnica difiere de los diseños tradicionales de manejo de conflictos, como intervención que posibilita a los protagonistas de la desavenencia la oportunidad de dialogar sobre sus intereses y construir, con la ayuda del mediador, una solución mutuamente satisfactoria.

El primordial objetivo es evaluar la aplicabilidad de la mediación comunitaria en Brasil como instrumento de eficacia de la democracia participativa. Para concretizarlo, esta investigación fue estructurada en tres partes distintas. La primera aborda el sentido y las peculiaridades de la palabra comunidad. El segundo apartado, a su vez, trata de la mediación en la comunidad como herramienta de empoderamiento individual y social y, por fin, el tercer topico investiga la relevancia democratica de la Mediación Comunitaria en el escenario brasileño.

\section{El sentido de comunidad y sus peculiaridades}

Antes de analizar las peculiaridades de la mediación comunitaria en Brasil es fundamental definir un concepto para comunidad. La palabra comunidad tiene su origen en el término latino communitas. Su 
concepto, igual que muchos de los conceptos de las ciencias sociales, no es univoco ni tampoco universal. En verdad, la palabra comunidad es polisémica, puede ser utilizada en distintos contextos, pero existe un elemento común en todos sus sentidos: la referencia a la cualidad de común, sea de personas que forman parte de un pueblo, nación, o conjunto de países o simplemente de personas que tienen interés y modo de vivir comunes.

Ferdinand Tönnies fue uno de los primeros sociólogos que se dedicó al estudio de la comunidad. En su obra Gemeinschaft und Gelellschaft, publicada en 1887, Tönnies desarrolla un estudio sobre la distinción de comunidad y sociedad, considerándolas dos formas opuestas de organización mutuamente excluyentes, que se basan sobre dos tipos de voluntad humana distintas: la voluntad "reflexionada" y la voluntad "orgánica." Tönnies enumera tres tipos de comunidades que se forman sobre: la base de las relaciones parentales, de vecindario o de amistad.

Así, el concepto de comunidad desarrollado en el siglo XIX mantenía relación con el sentido de proximidad geográfica que proporcionaba vínculos más estrechos entre los individuos. Las comunidades eran consideradas formas de asociación de diversas naturalezas que se oponían a la sociedad como forma de organización más amplia.

Los estudios sobre comunidad que se siguieron, desarrollado por pensadores considerados como clásicos en el tema, apuntan, cada uno a su modo, características bastante rigurosas para que determinado agrupamiento social fuese considerado una comunidad. Por supuesto, no existía y no existe en la actualidad consenso sobre las características fundamentales de comunidad.

Los estudiosos, principalmente sociólogos, fueran perfeccionando y añadiendo características que a su tiempo parecían necesarias para la determinación de una comunidad. Peruzzo resalta el conjunto de las principales condiciones básicas suscitadas por dichos estudiosos para la existencia de una comunidad: 
a) um processo de vida em comum por meio de relacionamentos orgânicos e certo grau de coesão social; b) auto-suficiência (as relações sociais podem ser satisfeitas dentro da comunidade, embora não seja excludente); c) cultura comum; d) objetivos comuns; e) identidade natural e espontânea entre os interesses de seus membros; f) consciência de suas singularidades identificativas; g) sentimento de pertencimento; h) participação ativa; i) locus territorial específico; e j) linguagem comum (PERUZZO, 2006, p. 13)

Estos rasgos configuran un tipo de comunidad tan perfecta, tan ideal, que sería difícil su existencia en las sociedades contemporáneas, especialmente las nociones de territorialidad, auto-suficiencia e identidad casi total como era concebida. Si vecindad y parentesco eran aspectos predominantes para definir una comunidad, hoy constituyen algunos de los muchos modelos posibles de las redes sociales.

Sin embargo, no se pueden despreciar las descubiertas por los clásicos, al contrario, hay que adecuarlas a la realidad actual y ampliar el concepto de comunidad a la moderna concepción de un mundo globalizado y tecnológico. Así, no es necesario que todos los elementos apuntados estén presentes, pero algunos de ellos todavía permanecen como elementos importantes para caracterizar las comunidades: sentimiento de formar parte de la comunidad, la interacción, carácter cooperativo, confluencia de intereses, la participación. Por tanto, el concepto de comunidad ha cambiado considerablemente de sentido. Bauman enfatiza que

Comunidad es una de esas palabras que producen una buena sensación: está bien tener una comunidad, estar en una comunidad. Y la sensación que transmite la palabra comunidad es buena porque todos los significados que conlleva prometen placeres y frecuentemente los tipos de placeres que a uno le gustaría experimentar pero que parece echar de menos. (BAUMAN, 2006, p.5)

Así, la comunidad es un lugar que transmite una buena sensación: que hacemos parte de algo mayor. Se trata de un lugar cálido, acogedor 
y confortable, que nos transmite confianza y seguridad. Pero este concepto de comunidad que habita nuestro imaginario es distinto de la comunidad que existe en la realidad.

La comunidad real que se presenta en el mundo global y multicultural que vivimos representa un espacio de lucha, de inseguridad. Para Bauman "se trata de un paraíso perdido o un paraíso que todavía se tiene la esperanza de encontrar" que nos remete al dilema de conciliar nuestra libertad individual con la vida en comunidad para que verdaderamente podamos vivir con seguridad. (BAUMAN, 2006, p.7)

Para Bauman, los conceptos de libertad y comunidad son incompatibles hoy en día. Existe un precio a pagar por el privilegio de vivir en comunidad que es justamente la libertad, la identidad. Cualquiera que sea la elección, habrá un beneficio y una pérdida. No tener comunidad significa no tener protección; por otra parte, alcanzar la comunidad, si es posible, podrá significar perder parte de la libertad. (BAUMAN, 2006, p.8)

Estamos delante de nuevas formas de asociaciones inmersas en una compleja red social con muchas posibilidades y dimensiones. El equilibrio entre libertad y comunidad es posible desde que no vislumbremos el modelo de comunidad establecida por los clásicos como el único viable. De hecho, es improbable la construcción de la comunidad ideal, completamente equilibrada, libre e igualitaria.

El capitalismo, la sociedad de consumo, la globalización, la tecnología y otros factores constituyen dificultades al proceso de construcción de comunidades adecuadas porque la masificación de las informaciones dificulta que las personas hagan sus elecciones de modo libre. Por eso, para disminuir los efectos derivados de estos procesos es imprescindible hacer los miembros de la comunidad conscientes, ciudadanos participativos que discuten sus decisiones, escuchan, defienden sus derechos y cumplen sus deberes.

En Brasil la palabra comunidad ha adquirido una característica especial. En los últimos años ha sido vinculada a las comunidades de 
baja renta, antes denominadas de favelas (barrio de chabolas). Esto no significa que el término no sirva para designar otros tipos de comunidad. Lo que pasa es que tanto en la vida cotidiana de la sociedad como en los medios de comunicación es cada vez más frecuente el uso de la palabra comunidad para sustituir la expresión favela.

Lo que parece es que este cambio se propone a mejorar, aunque artificialmente, la imagen de las favelas, cuya denominación conlleva una imagen peyorativa, de pobreza y sufrimiento. Los que viven en favelas son denominados favelados (chabolistas), otra palabra que tiene un sentido negativo, muchas veces vinculado a la marginalidad y criminalidad.

Essa concepção moral dos termos "favela" e "favelado" está associada ao estigma produzido historicamente em relação a estes espaços urbanos e seus habitantes, isto é, à concepção da favela enquanto espaço estigmatizante. Conforme indiquei no início do artigo, o próprio termo "favela" vem, ao longo do tempo, adquirindo um caráter depreciativo, uma vez que em torno desses espaços se construiu no imaginário social uma série de mitos ligados à pobreza, à violência e à criminalidade, que se refletiam nas ações do poder público neles empreendidas e na consequente estigmatização de seus habitantes como pobres, violentos e marginais em potencial (...). Visando disminuir esse estigma, a categoria "comunidade" parece evocar, tanto para os representantes do poder público quanto para os moradores diretamente atingidos pelo processo de estigmatização, uma alternativa simbólica viável. (FREIRE, 2008, p. 108-109)

En el seno de estas comunidades proliferan conflictos de distintas naturalezas. Las relaciones de interdependencia son cada vez mayores e intensas y por eso exigen la compresión mutua del otro. Vivir armoniosamente juntos presupone la necesidad de lograr soluciones inteligentes para los inevitables conflictos a través del diálogo pacífico. Así, para que podamos convivir en paz en la gran aldea planetaria globalizada debemos primero aprender a convivir en las comunidades a las cuales pertenecemos, que forman parte de nuestra vida cotidiana. 
Los conflictos en la comunidad son consecuencia de relaciones de tracto sucesivo, tienden a ser prolongados en el tiempo, convirtiéndose en graves problemas caso de que no sean solucionados en tempo hábil y de forma adecuada. "Así pues, si se consigue que los conflictos vecinales se solucionan en el propio ámbito comunitario, ya sea por negociación directa, ya sea por conciliación, ya sea por mediación, ello permite asegurar el mejor funcionamiento de la comunidad y garantizar su salud y coexistencia pacífica. (MEJÍAS GÓMEZ, 2009, p. 20)

\section{La mediación en la comunidad: empoderamiento individual y social}

La mediación comunitaria es la que más se ha destacado en el escenario brasileño. ${ }^{1}$ Esta modalidad de mediación ha sido desarrollada en Brasil como acción estratégica de democratización y facilitación del ejercicio del derecho fundamental de acceso a la justicia por parte de una parcela marginalizada de la población nacional, carente de instrumentos adecuados de prevención y solución de conflictos.

La necesidad de construir comunidades mediadoras es latente y fundamental en el contexto brasileño, caracterizado por una fuerte desigualdad social y por el alto índice de violencia. La aplicabilidad de la mediación en las comunidades del país, principalmente en los barrios más pobres, ha representado numerosas ventajas para los individuos, como forma de emancipación y empoderamiento; para la comunidad, como mecanismo de pacificación e inclusión social y para el conjunto de la sociedad, como instrumento de democracia participativa.

La mediación comunitaria ofrece un espacio para resolver los conflictos entre los individuos, grupos y organizaciones de la comunidad en la que se desarrolla. Su gran virtud es que

Sobre las experiencias de mediación comunitaria en el país, ver las obras de Lilia Maia de Morais Sales Comunidade".

"Justiça e mediação de conflitos" y "Mediação de Conflitos: Família, Escola e 
O método da mediação põe a comunidade na situação de protagonista da solução dos conflitos. Pela mediação a comunidade aprende a lidar com técnicas de comunicação construtiva e de relações interpessoais, absolutamente necessárias ao aprimoramento das suas ações, quer em relação ao conflito, quer no tocante à emancipação e desenvolvimentos sociais. (VASCONCELOS, 2008, p. 111)

Comunicación y mediación aparecen así como dos dimensiones necesariamente interrelacionadas. (RIQUELME, 2009, p. 145) La comunicación es la base de la mediación y está orientada para conseguir el consenso, la decisión pactada, celebrada mediante el acuerdo de voluntad de las partes en conflicto. Desarrollar habilidades de comunicación es fundamental en las comunidades porque sus miembros necesitan en todo instante negociar sus diferencias, compatibilizar sus intereses. Así, estimular una comunicación adecuada

Desenvolver essas qualidades é uma questão de sobrevivência para as comunidades de baixa renda, envolvidas em uma cultura de violência de feição coercitiva e piramidal, incompatível com a nova sociedade dos conhecimentos em franca expansão, em que se faz necessária a habilidade para lidar com relações horizontais, fundadas na persuasão e na negociação. (VASCONCELOS, 2008, p. 111)

Los índices de violencia en Brasil son muy altos, principalmente en las comunidades menos favorecidas. Existe una intensa red social que genera numerosos conflictos, donde la inseguridad y la creciente violencia en todos sus niveles torna urgente la necesidad de articular las relaciones interpersonales y las relaciones entre los individuos y el conjunto de la sociedad, para incentivar el diálogo y el respeto a las diferencias.

En estas comunidades se desarrollan conflictos peculiares y recurrentes, que son consecuencia de una vida difícil, marcada por la ausencia de una educación de calidad y carencia económica. En este contexto, es preciso cultivar una comunicación eficaz, solidaria e 
inclusiva para que la violencia no siga siendo utilizada para resolver las disputas.

La mediación comunitaria atiende a esta necesidad y por eso representa un instrumento de pacificación social. Pacifica porque enseña a las personas a asumir posturas pacíficas y no violentas delante de los conflictos con el otro. Las personas son incentivadas a escuchar, a pensar en el otro además de pensar en sí mismos. De esa manera, la violencia, sea física o psicológica, va siendo sustituida por soluciones amigables, que no sólo resuelven el problema puntual sino también son capaces de reestructurar las relaciones interpersonales.

La utilización de la mediación en las comunidades sobrepasa la satisfacción de intereses individuales y mejora la convivencia social. Fortalece el sentimiento de comunidad y permite a sus miembros sentir que forman parte de algo mayor.

Esta modalidad de mediación también promueve inclusión social. Esta inclusión es consecuencia del tratamiento dispensado a las partes, que pueden resolver por ellas mismas sus conflictos, de acuerdo con sus propios intereses. Pasan a tener voz activa, a desarrollar una consciencia crítica, transformándose en seres autónomos. Para Habermas:

Sob as condições de una compreensão pós-metafísica do mundo, só tem legitimidade o direito que surge da formação discursiva da opinião e da vontade dos cidadãos que possuem os mesmos direitos. Estes, por seu turno, só podem perceber, de maneira adequada, sua autonomia pública, garantida através de direitos de participação democrática, na medida que sua autonomia privada for assegurada. Uma autonomia privada assegurada serve como garantia para a emergência da autonomia pública, do mesmo modo que uma percepção adequada da autonomia pública serve como garantia para a emergência da autonomia privada. (HABERMAS, 2003, p. 146)

Una de las consecuencias del reconocimiento de la autonomía personal es el empoderamiento de la población. Existen grupos que están al margen del poder y necesitan de apoyo para empoderarse. 
El empoderamiento está relacionado con un cambio positivo en los individuos, cuyas consecuencias provocan cambios en la estructura y en la organización de las respectivas comunidades. Este proceso objetiva disminuir el desequilibrio existente y aumentar el poder de los grupos más débiles para que ellos puedan disfrutar mejor del acceso al poder formal e informal en su convivio social.

El empoderamiento también deriva de la divulgación más amplia de los derechos y deberes de las personas. En las comunidades pobres existe un déficit de conocimiento sobre las reglas del ordenamiento jurídico vigente y por eso es importante que los individuos participen de una sesión de mediación bien estructurada porque eso facilita a que ellos se vuelvan más conscientes y politizados.

El empoderamiento no es simplemente una terapia para que los pobres sean mejores con su pobreza tampoco es simplemente un apoyo a las iniciativas locales para que desarrollan una consciencia política más agudizada. Existe el reconocimiento de que las personas no están totalmente destituidas de poder y que existen redes previas de solidaridad y resistencia a través de las cuales la comunidad y sus individuos se organizan para defender sus intereses. La propuesta de empoderamiento está relacionada con un cambio positivo en estas personas y en sus respectivas comunidades para perfeccionar su estructura, organización y capacidad de establecer negociaciones positivas. (OAKLE; CLAYTON, 2003, p. 12-13)

Además del empoderamiento individual, la mediación también promueve el empoderamiento colectivo, de la comunidad como un todo, que pasa a reconocer el poder comunitario de transformar la realidad social y prevenir la violencia. Como consecuencia natural, el grupo tiende a adoptar un comportamiento positivo frente a los problemas comunes y a los conflictos interpersonales, y facilita la adopción de un abordaje creativo para la solución de conflictos relacionados con miembros de la comunidad.

La mediación comunitaria es una práctica y un proceso de creatividad social. No estamos hablando de la creatividad 
en relación con momentos extraordinarios o únicos, sino de manifestaciones vitales de nuestra existencia que están vinculados a construir formas y estilos de vida que los construimos desde la vida cotidiana. Desde los espacios de comunalidad, de encuentro, de interrelación. La mediación busca la conquista o la ocupación del espacio público como lugar de expresión de la sociedad civil. De sujetos con igual legitimidad política que quieren participar y dialogar a través de la palabra o de cualquier otra manifestación creativa. Es una práctica emergente, instituyente, transformadora y que permite la construcción de la ciudadanía. (MUSCOLO, 2008, p. 130)

En consecuencia, se plantea un nuevo paradigma que presupone soluciones creativas para los conflictos como una necesidad de las sociedades posmodernas. En ese contexto, el diálogo colaborativo, la espontaneidad, la innovación y la flexibilidad pasan a ser elementos fundamentales para dar respuestas satisfactorias a nuestras disputas. Esta energía creativa y mediadora puede ser utilizada por todos en cualquier contexto relacional, independientemente de su clase social, en cuanto no infrinja al ordenamiento jurídico vigente. Por tanto, "la mediación no solo puede ser una herramienta de intervención, sino que debe confundirse como una verdadera filosofía social, que persigue la cohesión comunitaria, la comunicación constructiva, la relación personal y la integración social." (MUNNÉ, 2006, p. 66)

Es importante advertir que cuando se habla de cohesión no significa homogeneidad o ausencia de conflictos en la comunidad, al contrario, la mediación reconoce el aspecto natural y la potencialidad transformadora de los conflictos, al mismo tiempo que reconoce cada individuo que forma parte de una comunidad como un ser único, cuyos intereses e sentimientos deben ser respetados.

Constituye una fuente de legitimación indiscutible la posibilidad de que los individuos tengan un espacio para dialogar y que este intercambio esté rodeado de atención y respeto para la palabra de cada uno. El espacio abierto para la discusión del conflicto posibilita que un mediado hable de sus intereses y escuche la narrativa del otro. 
Ese proceso comunicativo no está orientado hacia a la uniformidad o homogenización. Al contrario, pretende enaltecer la individualidad de cada ciudadano y la comprensión mutua de dicha individualidad.

\section{Mediación Comunitaria y su relevancia democratica en el escenario brasileño}

El desafío de concebir comunidades mediadoras ya está siendo llevado a cabo en Brasil, no obstante la falta de ley específica para regular la mediación. ${ }^{2}$ Existen programas de mediación comunitaria implementados en distintas ciudades brasileñas, frutos de iniciativas de actores gubernamentales y privados que pretenden beneficiar determinada comunidad con el uso de la mediación. Cada propuesta tiene sus características propias y se adecúa a la realidad del respectivo entorno social.

Los tipos de conflictos más comunes que son resueltos a través de estos programas de mediación comunitaria son los conflictos familiares y entre vecinos, o sea, conflictos de naturaleza continuada, que dependen

2 Aunque no exista ley específica de mediación en Brasil, el Consejo Nacional de Justicia ha desarrollado un papel fundamental para incentivar el uso de la mediación. En 29 de noviembre de 2010 publicó la Resolución 125 que dispone sobre la Política Judicial Nacional de tratamiento adecuado de los conflictos de intereses en el ámbito del Poder Judicial e introduce los mecanismos consensuales, principalmente la mediación y la conciliación en las actividades de la administración de justicia. El Consejo Nacional de Justicia ha desempeñado un papel fundamental en el desarrollo de esta política pues consiste en el órgano competente para: organizar acciones de incentivo a la autocomposición de los conflictos que deben tener la participación de todos los órganos del Poder Judicial e incluso la participación de entidades públicas y privadas asociadas, principalmente de las Universidades. El Consejo debe establecer las directrices para la implementación de la Política Nacional de tratamiento adecuado de los conflictos, determinando la competencia de los Tribunales; desarrollar el contenido mínimo y las acciones para la capacitaciones de los responsables de las mediaciones y conciliaciones y los otros facilitadores de la solución consensual de controversias; establecer que las actividades que envuelvan los mecanismos complementarios de solución de conflictos sean consideradas en las promociones de los magistrados a través del criterio de merecimiento; regular el Código de Ética correspondiente; batallar para que las instituciones de enseñanza divulguen estas prácticas con la creación de disciplinas sobre la materia, incluso en el curso de formación y perfeccionamiento de los magistrados; establecer la interlocución entre el orden de los abogados de Brasil y los demás órganos que forman parte de la administración de justicia en el sentido de incentivar la participación en la actuación y prevención de disputas. Sobre la Resolución 125 del CNJ ver: http://www.cnj.jus.br. Acceso en: 17.01.2014. 
de una solución eficaz para propiciar la convivencia armónica. De un modo general, las unidades de mediación son instaladas en el seno de las comunidades para facilitar el acceso de la población. El servicio es gratuito ya que pretende beneficiar las comunidades carentes. Los mediadores son voluntarios, personas de la propia comunidad o no, que son capacitados en mediación de conflictos. Los acuerdos logrados en estos proyectos están adquiriendo el progresivo reconocimiento por parte de la población, de los jueces y tribunales, que incluso han promovido la homologación y ejecución de estos acuerdos cuando es necesario.

Las prácticas de mediación comunitaria en Brasil han sido desarrolladas de modo independiente, sin que haya una sistematización entre las mismas. En cierto modo, eso es bueno porque posibilita que cada práctica sea pensada y ejecutada de acuerdo con su respectivo entorno. En contrapartida, sin criterios estandarizados, universalmente válidos para la capacitación y para el desarrollo de las actividades de los mediadores, es difícil garantizar la calidad de los servicios ofrecidos.

En consecuencia de todo lo anteriormente expuesto, la mediación debe ser entendida como pieza clave para la cultura política ciudadana porque proporciona una experiencia democrática más eficaz. Democracia y comunidad son elementos que caminan juntos, lado a lado, pues la idea de democracia presupone la vida en comunidad y viceversa. De acuerdo con John Dewey la democracia no es una alternativa a otros principios de vida asociativa, sino que es la propia idea de comunidad. "The clear consciousness of communal life, in all its implications, constitutes the idea of democracy." (DEWEY e outros, 1998, p. 295)

Así, la democracia tornase posible cuando tiene como punto de partida la comunidad y todos sus elementos constitutivos. Sustentase a partir del elemento social y no sólo del elemento político y por eso depende de la capacidad de autodeterminación de los individuos considerados individualmente y de la capacidad de autodeterminación de la comunidad. Por tanto, solamente existirá democracia cuando existieren comunidades donde las personas puedan verdaderamente ser respetadas y participar activamente de la vida en sociedad. 
En este sentido si no existe democracia en el nivel de la comunidad, no podrá existir democracia en ningún otro nivel. Ambas, democracia y comunidad, implican la participación activa de sus ciudadanos.

La democracia en Brasil es reciente (1988) y todavía no está efectivamente consolidada pues existen una serie de limitaciones de orden ideológico, político, económico, cultural, técnico, e incluso psicológico, que deben ser superadas. La democracia brasileña carece de la utilización de instrumentos de participación popular que puedan minimizar el inmenso abismo que separa a los ricos y los pobres, que permanecen al margen de la sociedad y encuentran numerosas dificultades para gozar de sus derechos.

Por tanto, el modelo que se plantea no es más el modelo de democracia representativa simplemente sino el modelo de democracia participativa. "El Concepto de democracia participativa aparece de alguna manera enfrentado al de democracia representativa, como la expresión de una nueva forma de democracia directa que amplia respecto de la democracia representativa los espacios de representación y participación de la ciudadanía dándole un rol más protagónico en el proceso deliberativo." (BIN, 2004, p. 22)

En la democracia participativa los ciudadanos deben deliberar y no sólo elegir sus representantes para que estos asuman todas las importantes decisiones que repercutirán en la sociedad. Asimismo, la ciudadanía debe participar directamente en el ejercicio y control de la fiscalización de las funciones públicas.

De acuerdo con Santos existen dos formas posibles de combinación entre democracia participativa y representativa: coexistencia y complementariedad. (SANTOS, 2001.) La coexistencia presupone una convivencia de las más variadas formas de procedimientos e instituciones; la democracia representativa en nivel nacional coexiste con la democracia participativa desarrollada en el nivel local. La complementariedad, a su vez, presupone una relación más intensa entre las dos formas de democracia: representativa y participativa. Aquí el gobierno debe reconocer que la participación activa es imprescindible 
al ejercicio de la democracia y por eso debe promover la utilización de prácticas que preconicen la participación de la sociedad como un todo.

Por supuesto, esta complementariedad entre las dos formas de democracia es fundamental para el verdadero ejercicio de la democracia una vez que la modalidad representativa, por si sola, es incapaz de viabilizar la democracia como sistema. Santos establece tres fases para el fortalecimiento de la democracia participativa:

Tesis 1:Através del reconocimiento de que no existe una única forma para la democracia. Al contrario, el multiculturalismo y las experiencias recientes de participación indican múltiples formas de deliberación pública. Así, el primer elemento de la democracia participativa consiste en la profundización de los casos en que el sistema político abre la mano de sus prerrogativas decisorias a favor de instancias colectivas de decisión.

Tesis 2: A través del fortalecimiento de la articulación contrahegemónica entre lo local y lo global. La articulación global/local torna posible el aprendizaje continuo, considerado requisito fundamental para el éxito de las prácticas democráticas de alta intensidad.

Tesis 3: Ampliación del experimentalismo democrático. La pluralización cultural, racial y distributiva de la democracia requiere que se multipliquen los experimentos en estas direcciones. Las experiencias de democracia participativa rescatan la dimensión pública y ciudadana de la política en la medida en que crean formas alternativas de organización del sistema político y realizan, desde esta perspectiva, una redimensión global de la vida colectiva. (SA NTOS, 2002, p.49)

Según lo dicho, se hace evidente que la pluralización de las modalidades de participación de toda la sociedad, sin exclusiones, sin permitir que algunas subjetividades prevalezcan sobre las otras, es una perspectiva interesante para los países que están consolidando sus experiencias democráticas.

No existe una secuencia lógica para el conjunto de luchas para hacer efectiva la democracia participativa. En las dos últimas décadas 
se han registrado en Brasil avances en la construcción de dicha democracia, con la implementación de numerosos instrumentos que permiten el incremento de la participación popular. Podemos citar por ejemplo la utilización de la mediación comunitaria. En este proceso es evidente la influencia del cambio producido en la sociedad civil:

Cambio que se expresa no solo en la exteriorización de la sensación de malestar por la pérdida del bienestar general, sino también en el fortalecimiento de los nuevos movimientos sociales ante el debilitamiento y retroceso del Estado para atender la demanda social, fenómeno que ha ido aumentando significativamente desde mediados de la década del ' 80 y que ha coincidido con las apertura de los procesos de democratización en América Latina. (BIN, 2004, p. 23.)

Los cambios significativos en la sociedad civil culminaron en el fortalecimiento del tercer sector que pasó a articular distintos movimientos y actores sociales para asumir la responsabilidad de representar y defender una multiplicidad de intereses de diversos sectores de la sociedad. Los nuevos movimientos sociales exigieron la participación activa de la ciudadanía, frente al déficit de satisfacción con el poder estatal del país, cuya trayectoria clientelista, patrimonialista y corrupta poco representaba el interés de la masa.

En este escenario, se fortalece la ciudadanía y se abren las puertas de un modelo democrático que se extiende a las instancias deliberativa y decisoria. De esa manera, se introduce una nueva consciencia ciudadana en la medida en que capacita, responsabiliza e informa la sociedad, lo que es fundamental para un Estado Democrático. La implementación de mecanismos participativos a nivel local, en el barrio, en la comunidad representa un verdadero cambio de paradigmas ya que tradicionalmente los individuos han esperado que el Estado y sus distintos poderes presenten una respuesta eficaz a sus demandas, lo que no ha sido plenamente posible.

La mediación comunitaria se presenta como alternativa viable para contribuir a la superación del problema de acceso a la justicia en 
Brasil porque propone una amplia revolución democrática del derecho y de la justicia. La revolución democrática de la justicia es una tarea compleja y exigente que debe partir de una concepción emancipadora del acceso al derecho y a la justicia.

En este sentido, la mediación comunitaria promueve una mayor democratización del acceso a la justicia, en sentido amplio de justicia, una vez que se permite a las partes formular un acuerdo justo según sus intereses. "Justa porque ambas partes han considerado que esa es la mejor solución que han alcanzado, de forma pacífica, cediendo ambas, de manera equivalente, en sus pretensiones iniciales para alcanzar un punto final mutuamente satisfactorio y que ambas se comprometen a cumplir de forma voluntaria." (MEJÍAS GÓMEZ, 2009, p. 11.)

El problema del acceso a la justicia en Brasil es histórico y remonta a la falta de participación popular en momentos cruciales de la trayectoria del país. La escasa participación de los ciudadanos incrementó la costumbre de permanecer pasivo, esperando una decisión del Estado. Por eso, para posibilitar la revolución jurídica tan deseada, es fundamental incentivar prácticas de movilización social, construidas socialmente en el seno de las comunidades, con la participación activa de los individuos.

Para una eficacia todavía mayor del derecho fundamental de acceso a la justicia en Brasil sería muy beneficiosa la inclusión de un sistema "multipuertas" para ofrecer formas hibridas de solución de conflictos para que las partes puedan elegir el mejor método de solución para su caso. En otras palabras, sería beneficioso reconstruir el panorama de la administración de justicia en el país para contemplar los modernos instrumentos de acceso a la justicia. "A realidade social aponta para a necessidade de corte das amarras de um direito legalista, textualizado, estatal, e da quebra de paradigmas de elaboração, interpretação e aplicação do direito." (BEZERRA, 2008, p. 185.)

En resumen, la gestión democrática participativa exige el entendimiento de cada espacio como un eslabón mediador que invita a los ciudadanos a la participación y a la implicación en este proceso, 
rompiendo con la visión paternalista y redentora del Estado, que ha predominado en la historia de Brasil.

En el contexto brasileño, marcado por la fuerte desigualdad social, la mediación comunitaria, ampliamente utilizada en las "favelas", o comunidades de baja renta, permite el enfrentamiento de cuestiones que envuelven la exclusión social, la desigual distribución de poder y la violación de derechos humanos, factores negativos que alimentan la violencia y dificultan la real efectividad de la democracia.

\section{Conclusión}

La estructura social, económica y jurídica de Brasil está pasando por profundas transformaciones pero todavía queda un largo camino hasta que el país se consolide como un efectivo Estado Democrático, una vez que la eficacia de los derechos fundamentales, sobre todo para los pobres, choca en numerosos obstáculos.

La gestión democrática participativa debe invitar a los ciudadanos a la participación y a la implicación en el proceso de toma de decisiones, rompiendo con la visión paternalista y redentora del Estado, que ha predominado en la historia de Brasil.

La mediación comunitaria coincide con estos valores porque genera una mayor responsabilidad y participación de la comunidad en la solución de sus conflictos y concibe ciudadanos más conscientes. A través de la comunicación, construye relaciones más cooperativas y conduce a la comunidad para una transformación socio-cultural positiva, orientada hacia el ejercicio activo de la ciudadanía y, consecuentemente de la democracia.

En el contexto brasileño, marcado por la fuerte desigualdad social, la mediación comunitaria, ampliamente utilizada en las "favelas", o comunidades de baja renta, permite el enfrentamiento de cuestiones que envuelven la exclusión social, la desigual distribución de poder y la violación de derechos humanos, factores negativos que alimentan la violencia y dificultan la real efectividad de la democracia. 
No obstante la falta de ley específica sobre mediación, las experiencias prácticas se multiplican en el país, principalmente con la modalidad de mediación comunitaria. La aplicabilidad de la mediación en las comunidades, sobre todo en las más pobres, extrapola la satisfacción de los mediados y afecta la sociedad de un modo general. Esto porque desarrolla en la población valores, comportamientos favorables al fortalecimiento de una cultura de paz, basada en el diálogo y en la tolerancia. La mediación comunitaria es realizada en el seno de estas comunidades, y por eso crea espacios públicos de discusión, de experiencia democrática.

\section{Referências}

BAUMAN, Z. Comunidad: en busca de seguridad en un mundo hostil. Trad. de J. Alborés. Madrid: Siglo XXI, 2006.

BEZERRA, Paulo Cesar Santos. A produção do Direito no Brasil. 2. ed. Bahia: Editus, 2008.

BIN, Elisa. Algunas reflexiones para el debate acerca de la construcción de la Democracia participativa. En: ROMERO, Ricardo (Comp.). Democracia participativa, una utopía en marcha. Buenos Aires, 2004.

DEWEY, John. HICKMAN, Larry; ALEXANDRE, Thomas M. (Ed.). The Essential Dewey: Pragmatism, education y democracy. Bloomington: Indiana University Press, 1998.

FREIRE, Leticia de Luna. Favela, bairro ou comunidade? Quando uma política urbana torna-se uma política de significados. 2008. Disponible en: http://revistadil.dominiotemporario.com. Acceso em: 15. marzo 2014.

HABERMAS, Jurgen. Direito e democracia: entre facticidade e validez. 2. ed. Tradução de Flávio B. Siebeneichler. Rio de Janeiro: Tempo Brasileiro, 2003, vol. II.

HIGHTON, Elena. Justicia en cambio: sociedad civil, abogados y jueces. Un nuevo proyecto para la administración de justicia. Buenos Aires, 2003. 
MEJÍAS GÓMEZ, Juan Francisco. La mediación como forma de tutela judicial efectiva. Madrid: El derecho y quantor, 2009.

MUNNÉ, María; MC-CRAGH, Pilar. Los 10 principios de la cultura de Mediación. Barcelona: Graó, 2006.

CARAM, María Elena; et al. Mediación: diseño de una práctica. Buenos Aires: Librería Histórica, 2006.

MUSCOLO, Silvia lannitelli, et al. La mediación comunitaria como práctica de creatividad social y de construcción de ciudadanía. En: Documentación Social: la mediación: caja de herramientas ante el conflicto social. Madrid: Cáritas Española, 2008.

OAKLEY, Peter; CLAYTON, Andrew. Monitoramento e avaliação do empoderamento. São Paulo: Instituto Polis, 2003.

PERUZZO, Cicilia Maria Krohling. Comunidades em tempo de redes. Disponible en: http://www.ciciliaperuzzo.pro.br. Acceso en: 07 abr. 2014.

RIQUELME, Sergio Fernández. Teoría y práctica de la mediación: la gestión alternativa de los conflictos sociales. Murcia: Edit.um, 2009.

SALES, Lilia. Justiça e mediação de conflitos. Belo Horizonte: Del Rey, 2004.

- Mediação de conflitos: família, escola e comunidade. Florianópolis: Conceito, 2007.

SANTOS, Boaventura de Sousa. Democratizar a democracia: os caminhos da democracia participativa. Rio de Janeiro: Civilização Brasileira, 2002.

- Pela mão de Alice: o social e o político na pós-modernidade. 8. ed. São Paulo: Cortez, 2001.

VASCONCELOS. Carlos Eduardo de. Mediação de conflitos e práticas restaurativas: modelos, processos, ética e aplicações. São Paulo: Método, 2008.

Recebido em: 04/09/2014

Aprovado em: 19/10/2014 